\title{
DIABETES IS PRIMARY TIMELY NEWS AND NOTES FOR PRIMARY CARE PROVIDERS from the American Diabetes Association
}

\section{FROM THE JOURNALS}

By Max Bingham, PhD

\section{Poor Adherence Cuts Real-World Effectiveness of Diabetes Drugs}

In the past decade, numerous treatment options for type 2 diabetes have received regulatory approval. However, survey data from the United States and elsewhere suggest that only about half of patients actually achieve the general $\mathrm{A} 1 \mathrm{C}$ target of $<7.0 \%$ despite receiving approved treatments that randomized, controlled trials (RCTs) have found to be effective. Are the trials wrong? Or, is something stopping the drugs from working as intended in the real world?

The latter appears to be the case. Carls et al. (Diabetes Care http://doi.org/cfq8) report that two sets of commonly used diabetes drugs were significantly less effective when taken by patients in the real world than when they were taken during RCTs. The reason? A full three-fourths of the gap could be explained by one factor: poor medication adherence.

The authors say that, although the difference they found in effectiveness is not surprising, the reason they identified for it highlights an urgent need to review strategies to improve adherence to achieve optimal health outcomes.

In an accompanying "Perspectives in Care" article (Diabetes Care http://doi.org/cfq9), Edelman and Polonsky, two of the study's authors, examine the implications of poor medication adherence and the discrepancies between findings in clinical trials and real-world outcomes. They say that it all points toward the under-realization of potential benefits from U.S. Food and Drug Administration (FDA)-approved drugs for the treatment of diabetes.

Poor medication adherence is widely recognized as a key health care issue in the United States and abroad. Edelman and Polonsky write that innovative approaches are "sorely needed" to effectively deal with this problem in clinical practice. They emphasize, however, that addressing poor adherence will be difficult because it is

Max Bingham, PhD, is a science writer and editor in Rotterdam, Netherlands. He can be reached on Twitter at @maxbingham.

https://doi.org/10.2337/cd17-0123

(C)2018 by the American Diabetes Association, Inc. not explained by a single behavior and thus will probably defy any one-size-fits-all solution.

Meanwhile, a meta-analysis by Khunti et al. (Diabetes Care http://doi.org/cfrb) suggests that good medication adherence likely reduces the risk of all-cause mortality and hospitalization in patients with type 2 diabetes. Their analysis of eight separate observational studies involving $\sim 320,000$ patients found a mean rate of poor adherence of $37.8 \%$ and relative risks for all-cause mortality and hospitalization of 0.72 and 0.90 , respectively, for patients with good and poor medication adherence.

We are reminded that, way back in 1985, then-U.S. Surgeon General C. Everett Koop bluntly summed up the issue of adherence as follows: "Drugs don't work in patients who don't take them."

\section{In the News: Soaring Rates of Obesity in Children}

Timed to coincide with World Obesity Day, The Lancet released an analysis (http://doi.org/cfrc) by the NCD Risk Factor Collaboration (www.ncdrisc.org) of obesity rates around the world, showing that, in the past 40 years, there has been a tenfold increase in the number of children with obesity. Specifically, there were $\sim 50$ million obese girls and 74 million obese boys worldwide in 2016. There were also hundreds of millions of adults with obesity and potentially as many as 1.3 billion in the overweight category.

Numerous experts used the widespread media reporting of these findings as an opportunity to call for improvements in diets and lifestyles to reduce obesity rates and to urge the food and pharmaceutical industries, governments, and health care providers to do more to combat obesity. A much less reported finding in the analysis was that there are still many more undernourished children globally than obese children, with rates of undernourishment remaining mostly stable since 1975. Nevertheless, the authors of the report suggest that, if current increases in obesity continue, there will apparently be more obese than malnourished children by 2022.

Released at the same time as the analysis in The Lancet, the World Obesity Forum published data (http://bit.ly/2h2ogUP) showing that the annual costs 


\section{\begin{tabular}{l|l}
$\begin{array}{c}\text { American } \\
\text { Diabetes } \\
\text { Association. }\end{array}$ & $\begin{array}{l}\text { Diabetes } \\
\text { Is Primary }\end{array}$ \\
\end{tabular}}

of dealing with obesity-related illness, including diabetes, will likely hit $\$ 1.2$ trillion by 2025 if current approaches continue.

In other reports, we learn that, in 2016 in Scotland (http://bit.ly/2zXOWeT, with a useful summary at http://bbc.in/2gSvxmo), about two-thirds of the population were overweight or obese, with around half of these being obese. Meanwhile, in the United States, obesity rates were nearly $40 \%$ in adults and $20 \%$ in children in 2015-2016, according to data from the Centers for Disease Control and Prevention (http:///bit.ly/2z5gYmp).

\section{New Analyses: Diabetes-Related Mortality in Germany and Diabetes Rates in China}

An analysis of diabetes-related mortality rates in Germany suggests that, in 2010, 174,627 excess deaths were caused by diabetes, with the majority due to type 2 diabetes. This equated to $21 \%$ of all deaths in Germany that year. Jacobs et al. (Diabetes Care http://doi.org/cfrf) say their estimates suggest that official figures based on death certificate information "grossly underestimate" mortality due to diabetes in the country (currently reckoned to be $2.3 \%$ ). They also say that their method, which is based on nationwide health care and insurance data, could be used to calculate mortality due to diabetes in other countries.

Meanwhile, an analysis by Li et al. (Diabetes Care http://doi.org/cfrg) suggests that China's ever-expanding rates of type 2 diabetes are being driven by BMI, unhealthy diets packed with refined grains, and, to some extent, low physical activity and high blood pressure. With the country accounting for 100 million diabetes cases (one-fourth of the world's total), each of the implicated factors likely accounts for millions of diabetes cases. For example, the authors estimate that $\mathrm{BMI}$ increases alone are responsible for 44 million cases. As author Frank B. Hu told Diabetes Care in its December 2017 issue, "Diabetes has become a public health crisis that also threatens to reverse economic gains made in recent decades in China. To curb this epidemic, prevention of chronic diseases such as diabetes through promotion of a healthy diet and lifestyle should be elevated to a national and global public policy priority."

\section{TREATMENTS + THERAPIES}

\section{Semaglutide: Injectable Formulation on}

Track for Approval, Success Reported

With Oral Formulation, and Efficacy as Obesity Therapy Explored

The injectable, once-weekly formulation of semaglutide, Novo Nordisk's glucagon-like peptide 1 (GLP-1) receptor agonist for type 2 diabetes, has received the nod from an FDA advisory committee, putting the drug on course for FDA approval (http://bit.ly/2zWUjZW). The Endocrinologic and Metabolic Drug Advisory Committee concluded that the drug is effective, carries no heart health risk, and poses only limited risk for sight problems. However, some members of the committee reportedly urged follow-up studies on the risk of diabetic retinopathy potentially associated with the drug.

Based on various trials in Novo Nordisk's SUSTAIN research program, semaglutide can reportedly lead to meaningful reductions in both $\mathrm{A} 1 \mathrm{C}$ and weight, outperforming both placebo and, significantly, its key competitor drugs sitagliptin (a dipeptidyl peptidase-4 inhibitor), insulin glargine, and once-weekly exenatide (another GLP-1 receptor agonist). Hints from the SUSTAIN-6 cardiovascular outcomes trial additionally point to heart health benefits. However, the drug can reportedly cause a variety of gastrointestinal complaints, and the FDA committee did zero in on possible links to diabetic retinopathy. On the plus side, the drug likely has low risk for hypoglycemia. A full report on the advisory committee meeting's outcomes can be found at MedPageToday (http://bit.ly/2/1oBrU).

Meanwhile, a once-daily oral form of the semaglutide being developed for the treatment of type 2 diabetes reportedly works as well as its once-weekly injectable formulation for both glycemic control and weight loss. Reporting in the Journal of the American Medical Association (http://doi.org/cfrt), Davies et al. write that, from baseline to week 26 of their trial, A1C decreased by $0.7-1.9 \%$ (depending on dosage) with oral semaglutide, $1.9 \%$ with injectable semaglutide, and $0.3 \%$ with placebo. Weight reductions with oral semaglutide ranged from 2.1 to $6.9 \mathrm{~kg}$ (again, dose-dependent), compared to reductions of $6.4 \mathrm{~kg}$ with injectable semaglutide and $1.2 \mathrm{~kg}$ with placebo. In terms of adverse events, a majority of patients in all groups reported mild to moderate gastrointestinal issues. The authors conclude: "Among patients with type 2 diabetes, oral semaglutide resulted in better 


\section{MARKETPLACE}

\section{Patients Referred to Weight Management Classes} Might Reduce Their Risk of Type 2 Diabetes

Obese patients at risk for diabetes who are referred to a diabetes prevention program (DPP) delivered through a commercial weight management provider might well be able to reduce their risk of progressing to the disease. As reported by Piper et al. (BMJ Open Diabetes Research and Care http://doi.org/cfrh), the intensive lifestyle modification program that combined a structured 90-minute activation session on diabetes prevention plus 48 weeks of standard Weight Watchers community group meetings reportedly helped about one-third of obese patients who took part in the study to achieve normal glucose levels and reduce their risk of diabetes. And, according to the authors, whereas most patients either reduced or at least did not increase their risk, only $3 \%$ developed type 2 diabetes by 12 months after starting the program. According to the authors, this outcome provides some of the first evidence from the United Kingdom's National Health Service (NHS) DPP and suggests that a primary care referral route for such intervention is likely to be effective. They conclude, "The lifestyle changes and weight loss achieved in the intervention translated into considerable reductions in diabetes risk, with an immediate and significant public health impact."

The NHS is planning to roll out its DPP referral program to millions of overweight people in the United Kingdom over the next few years, based on the notion that costs it incurs for patients to participate will be offset through savings from the prevention of diabetes. Diabetes medical care currently accounts for just under 9\% of the annual NHS budget. More about the NHS DPP can be found at http://bit.ly/2gopNR2.

\section{Artificial Pancreas Succeeds, But What Drives Uptake of the Technology?}

An artificial pancreas combination running on smartphone and Cloud-based algorithms has been successfully tested in patients with type 1 diabetes. According to Dassau et al., who reported the trial (Diabetes Care http://doi.org/cfrr), the system reduced $\mathrm{A} 1 \mathrm{C}$ from an average 7.0 to $6.7 \%$ during 12 weeks of use. Also, the amount of time spent in a glucose range indicative of hypoglycemia decreased from 5.0 to $1.9 \%$ during the day and from 4.1 to $1.1 \%$ at night over the 12 weeks of the study. Reportedly, the algorithms included in the system are improved versions of ones the authors have previously reported and are designed to control both insulin levels and carbohydrate ratio profiles.

"This is by far the longest duration trial we have conducted, and it is a testament to the robustness of the algorithm that our key indices were maintained from our earlier, shorter trials," author Frank Doyle said in a press release (http://bit.ly/2xAFIm8). "We took a disciplined group that had very good overall $\mathrm{HbA}_{\mathrm{lc}}$ levels before the trial, and we brought the group even lower."

Meanwhile, a qualitative study (Diabetes Care http://doi.org/cfrs) of users' expectations of automated insulin delivery systems (i.e., artificial pancreas systems) reveals a number of themes/ concerns that patients with type 1 diabetes and their caregivers might face when deciding to use the technology. Based on structured interviews and focus groups, Naranjo et al. report that children and adolescents with type 1 diabetes primarily identified needs based on their life stage and social context (e.g., school). Adults with type 1 diabetes were most concerned with more practical aspects of the technology, such as its accuracy, reliability, and potential ability to stabilize glucose levels and reduce the risks of complications. The authors say that these insights will hopefully assist researchers and clinicians in understanding why uptake of these devices may be limited.

Finally, the FDA has approved Abbott's FreeStyle Libre Flash Glucose Monitoring System (http://bit.ly/2yut0FV). This system has the notable feature of not requiring users to routinely prick their fingers to perform device calibrations with a traditional blood glucose meter. Instead, the device uses a patch with a small sensor wire that is inserted below the skin. Users can obtain a glucose reading by holding a dedicated mobile reader above the patch. According to the FDA statement, the device is intended for people with diabetes who are 18 years of age or older. After a 12-hour start-up period, it can then be worn continuously for up to 10 days.
Semaglutide, continued from p. 9

glycemic control than placebo over 26 weeks. These findings support phase 3 studies to assess longer-term and clinical outcomes, as well as safety."

Rounding off with a much smaller trial, Blundell et al. (Diabetes, Obesity and Metabolism http://doi.org/f9tb6z) report that once-weekly injectable semaglutide given to obese patients results in weight loss of $\sim 5 \mathrm{~kg}$ over 12 weeks, likely caused by a reduction in food intake by about one-fourth. Specifically, they say the drug decreases appetite and food cravings, leading to better control of eating behavior and a lower relative preference for fatty, energy-dense foods.
"What was striking was the potency of the drug's action," author John Blundell said in press coverage of the study (http://bit.ly/2IE5y7l). "We saw results in 12 weeks which may take as long as 6 months with other anti-obesity medication. The drug reduced hunger but also cravings for food and the sensation of wanting to eat, and these had previously been thought to stem from different parts of the brain."

A related study recently published in the same journal by Hjerpsted et al. (http://doi.org/cfrv) goes much more into the mechanisms that might be behind the apparent appetite-cutting ability of semaglutide. 


\section{ADA NEWS}

\section{INSULIN AFFORDABILITY}

The average price of insulin has skyrocketed in recent years-nearly tripling between 2002 and 2013. Insulin isn't optional. For millions of people living with diabetes, including all individuals living with type 1 diabetes, access to insulin is a matter of life and death. There is no medicine that can be substituted for insulin.

For more than a year, the American Diabetes Association (ADA) Make Insulin Affordable campaign has been bringing attention to this crucial issue. In 2016, the ADA board of directors issued a resolution calling on all entities in the insulin supply chain, including manufacturers, wholesalers, pharmacy benefit managers, insurers, and pharmacies, to substantially increase transparency in pricing associated with the delivery of insulin to individuals with diabetes. Transparency in this process is the first step to identifying viable long-term solutions for individuals with diabetes who are unable to afford the insulin they need. The resolution also calls on all entities in the insulin supply chain to ensure that no person with diabetes is denied affordable access to insulin.

As the organization whose mission is to improve the lives of all people affected by diabetes, ADA believes that no individual in need of lifesaving insulin should ever go without it due to prohibitive costs. To address this issue, the association has convened an Insulin Access and Affordability Working Group, which is working with multiple stakeholders at all levels of the insulin supply chain, people affected by the rising cost of insulin, and the U.S. Congress in an active effort to understand the underlying causes of the dramatic increase in insulin prices. The major objective of the working group is to bring about long-term solutions to ensure that insulin is affordable for all who need it. More information on these efforts is available from www.makeinsulinaffordable.org.

\section{THE USE OF LANGUAGE IN DIABETES CARE AND EDUCATION: A CONSENSUS REPORT}

Language has a strong impact on perceptions and behavior, and this may be especially true with regard to medical care and health-related behaviors. An ADA/American Association of Diabetes Educators joint task force has examined this issue with regard to diabetes care and education and developed recommendations for language used by health care professionals and others when discussing diabetes with patients, colleagues, or the general public. These recommendations, in addition to research questions related to language and diabetes, are outlined in a consensus report released simultaneously by Diabetes Care and The Diabetes Educator on 17 October 2017 (http://doi.org/cfrz).

To learn more about ADA's continuing education opportunities, including Diabetes Is Primary events in your community, please visit http://professional.diabetes.org/ce. 